\title{
THE REGULAR GROUP $C^{*}$-ALGEBRA FOR REAL-RANK ONE GROUPS
}

\author{
ROBERT BOYER AND ROBERT MARTIN ${ }^{1}$
}

\begin{abstract}
Let $G$ be a connected semisimple real-rank one Lie group with finite center and let $C_{\rho}^{*}(G)$ denote the regular group $C^{*}$-algebra of $G$. In this paper a complete description of the structure of $C_{\rho}^{*}(G)$ is obtained.
\end{abstract}

1. Introduction. Let $G$ be a connected semisimple real-rank one Lie group with finite center and Lie algebra $\mathrm{g}$. If $G_{\mathbf{C}}$ is the simply connected, complex analytic group corresponding to $\mathfrak{g}_{\mathbf{C}}$, we assume, in addition, that $G$ is the real analytic subgroup of $G_{\mathbf{C}}$ corresponding to g. Let $C_{\rho}^{*}(G)$ denote the regular group $C^{*}$-algebra of $G$, i.e., the completion of $L_{1}(G)$ with respect to the norm $\|f\|_{\rho}=\|\rho(f) \mid\|$ where $\rho$ is the left regular representation of $G$ and $\||\rho(f)|\|$ denotes the norm of $\rho(f)$ as a left convolution operator on $L_{2}(G)$. The purpose of this paper is to give a complete description of the structure of $C_{\rho}^{*}(G)$ and thus give a partial answer (one for the above $G$ ) to a question raised in [6] as to an intrinsic characterization of $C_{0}(\hat{G})$.

Throughout this paper $H$ will denote a fixed separable infinite-dimensional Hilbert space and $\mathscr{K}(H)$ will denote the compact operators on $H$. We assume, in addition, that $H$ has been identified with $H \oplus H$. When $T$ is a locally compact Hausdorff space, we denote by $C^{b}(T, \mathscr{K}(H))$ the $C^{*}$-algebra of all norm-continuous bounded functions $t \mapsto x(t)$ of $T$ into $\mathscr{K}(H)$ and by $C^{0}(T, \mathcal{K}(H))$ the $C^{*}$-algebra of functions in $C^{b}(T$, $\mathcal{K}(H))$ such that $\|x(t)\|$ vanishes at infinity.

The underlying hull-kernel topology on the spectrum of $C_{\rho}^{*}(G), \hat{G}_{r}$, plays a key role in describing the structure of $C_{\rho}^{*}(G) \approx C_{0}(\hat{G})$. The main difficulty occurs when $\hat{G}_{r}$ is not Hausdorff. When $\hat{G}_{r}$ is Hausdorff e.g.,

$$
G=\operatorname{Spin}(2 n+1,1) \text { for } n \geqslant 1,
$$

it follows from $[2,10.9 .6]$ that $C_{\rho}^{*}(G)$ is isomorphic to $C^{0}\left(\hat{G}_{r}, \mathscr{K}(H)\right)$. However, when $\hat{G}_{r}$ is not Hausdorff the above theorem no longer applies and we show, in $\S 3$, that it is possible to use the extension theory of $\mathrm{C}$. Delaroche [1] to determine the structure of $C_{\rho}^{*}(G)$. We first show that $C_{\rho}^{*}(G)$ is isomorphic to the restricted product of certain $C^{*}$-algebras whose structures

Received by the editors December 1, 1975.

AMS (MOS) subject classifications (1970). Primary 22D25, 43A40; Secondary 46L25.

Key words and phrases. Semisimple Lie groups, Cartan subgroups, Weyl group, irreducible unitary representations, left regular representation, principal series, discrete series, reduced dual, hull-kernel topology, $C^{*}$-algebras, restricted product.

${ }^{1}$ Partially supported by NSF-MPS 75-14490. 
have concrete descriptions given by [1, Theorem VI.3.8]. Letting $\mathscr{2}_{P}, \hat{G}_{P}$, and $\hat{G}_{d}$ be as in [8, Volume II], it is then a simple matter to give an alternate description of $C_{\rho}^{*}(G)$ as the subalgebra of functions in $C^{0}\left(\mathscr{Q}_{P} \cup \hat{G}_{d}, \mathscr{K}(H)\right)$ which reduce at the points of $\mathscr{Q}_{P}-\hat{G}_{P}$ (i.e., the points "responsible" for the non-Hausdorffness of $\hat{G}_{r}$ ) by $H \oplus H$.

We refer to [2] and [8] for all undefined terms and notation.

2. The topology on $\hat{G}_{r}$. In this section we summarize the main results concerning the representation theory of $G$ and the topology on $\hat{G}_{r}$ which we shall need to describe $C_{\rho}^{*}(G)$. For a more detailed account we refer to $[8$, Volume II, Chapter 7 and Epilogue].

Let $G=K A N$ be an Iwasawa decomposition for $G, M$ the centralizer of $A$ in $K, P=M A N$ (a minimal parabolic subgroup of $G$ ), and $W=\{1, w\}$ be the Weyl group of $G$, where $w$ is the unique nontrivial element of $W$. We let $\hat{G}_{r}$ denote the reduced dual of $G$ i.e., the support of $\rho$ in $\hat{G}$.

Up to conjugacy, either $G$ has a unique noncompact Cartan subgroup or $G$ has two Cartan subgroups-one compact and one noncompact. Each conjugacy class of Cartan subgroups makes its own contribution to $\hat{G}_{r}$. The noncompact Cartan subgroup contributes the collection of irreducible principal series representations, $\hat{G}_{P}$, together with those irreducibles which arise as summands of reducible principal series representations, $\check{G}_{P}$. The compact Cartan subgroup contributes the so-called discrete series of $G, \hat{G}_{d}$. Let us briefly recall the parameterizations of these representations given in [8].

If a denotes the Lie algebra of $A$, the irreducible unitary representations of $A$ are given by $\lambda^{s}(\exp H)=\exp (i s H), s \in \mathbf{R}, H \in$ a, and so $\hat{A}=\left\{\lambda^{s}\right.$ : $s \in \mathbf{R}\}$. The hull-kernel topology on $\hat{A}$ agrees with the usual topology it inherits as the character group of the abelian group $A$, i.e., that of $\mathbf{R}$. If $n$ is the dimension of a maximal torus in the compact group $M$, then we may view $\hat{M}$ as a countable discrete subset of $\mathbf{R}^{n}$ and, hence, $\hat{M} \times \hat{A}$ as a subset of $\mathbf{R}^{n+1}$ with the relative topology. The Weyl group $W$ acts on $\hat{M} \times \hat{A}$ as follows: $1 \cdot(\sigma, s)=(\sigma, s)$ and $w \cdot(\sigma, s)=(w \cdot \sigma,-s)$ where $w \cdot \sigma(m)=$ $\sigma\left(w^{-1} m w\right), \quad m \in M$. Under the quotient topology, the orbit space $\mathscr{Q}_{P}=$ $(\hat{M} \times \hat{A}) / W$ is locally compact and Hausdorff.

For $\sigma \in \hat{M}$ and $\lambda^{s} \in \hat{A}$ we form the finite-dimensional irreducible unitary representation $\sigma \times \lambda^{s}$ of $P$ via $\left(\sigma \times \lambda^{s}\right)($ man $)=\sigma(m) \lambda^{s}(a)$ and write

$$
\pi(\sigma, s)=\operatorname{Ind}_{P}^{G} \sigma \times \lambda^{s} .
$$

The collection of unitary representations $\{\pi(\sigma, s): \sigma \in \hat{M}, s \in \mathbf{R}\}$ is called the principal series of $G$. It is known that $\pi(\sigma, s)$ is irreducible unless $w \cdot \sigma=\sigma$ and $s=0$ and in this case $\pi(\sigma, 0)$ may or may not be irreducible (see [5] or [8, Volume I, p. 462]). When $G$ has a unique Cartan subgroup, the results of Wallach [7] show that every member of the principal series is irreducible. If we let $R=\{\sigma \in \hat{M}: \pi(\sigma, 0)$ is reducible $\}$ and $I=\hat{M}-R$, then for $\sigma \in R$ it is known that $\pi(\sigma, 0)$ decomposes into two inequivalent representations of $G$ (see [4]) which we shall denote by $\pi_{\sigma}^{ \pm}$. It follows from [8, 
5.5.3.3] that $\pi(\sigma, s) \simeq \pi\left(\sigma^{\prime}, t\right)$ iff there exists a $c \in W$ such that $c \cdot \sigma=\sigma^{\prime}$ and $c \cdot s=t$, i.e., $\sigma=\sigma^{\prime}$ and $s=t$ or $w \cdot \sigma=\sigma^{\prime}$ and $s=-t$. Thus we may identify the collection of principal series representations with $\mathcal{Q}_{P}$.

As in [8, Volume II], we let $\hat{G}_{P}$ denote the subset of $\mathscr{Q}_{P}$ consisting of irreducible principal series representations. A point $q \in \mathcal{Q}_{P}-\hat{G}_{P}$ has coordinates $(\sigma, 0)$ with $\sigma \in R$ and so we may associate to $q$ the pair of representations $\pi_{\sigma}^{ \pm}=\pi_{q}^{ \pm}$in $\hat{G}_{r}$ (it is this association that makes $\hat{G}_{r}$ non-Hausdorff). Letting $\check{G}_{P}=\left\{\pi_{q}^{ \pm}: q \in \mathcal{Q}_{P}-\hat{G}_{P}\right\}$ we have that $\hat{G}_{P} \cap \check{G}_{P}=\varnothing$.

When $G$ has a compact Cartan subgroup (iff rank $G \equiv 1+\operatorname{rank} M=$ rank $K)$, one obtains a family of irreducible, square-integrable, unitary representations of $G$ called the discrete series of $G$. Denoting this family by $\hat{G}_{d}$, we have that $\hat{G}_{d} \cap\left(\hat{G}_{P} \cup \check{G}_{P}\right)=\varnothing$ and that it is possible to parameterize $\hat{G}_{d}$ by a lattice in $\mathbf{R}^{n+1}[8,10.2 .4]$. Thus we may identify $\hat{G}_{d}$ with a countable discrete subset of $\mathbf{R}^{n+1}$ which does not intersect $\hat{G}_{P} \cup \check{G}_{P}$ and ultimately, $\hat{G}_{r}$ as a disjoint union of the three subsets $\hat{G}_{P}, \check{G}_{P}$, and $\hat{G}_{d}$ of $\mathbf{R}^{n+1}$.

TheOrem (Lipsman). Let $\hat{G}_{r}$ be the reduced dual of $G$. Then

(1) if $G$ has a unique Cartan subgroup, $\hat{G}_{r}=\hat{G}_{P}=\mathscr{Q}_{P}$ and the hull-kernel topology on $\hat{G}_{r}$ coincides with the natural (Hausdorff) topology of $\mathscr{2}_{P}$,

(2) if $G$ also has a compact Cartan subgroup, $\hat{G}_{r}=\hat{G}_{P} \cup \check{G}_{P} \cup \hat{G}_{d}$ (disjoint union) where both $\hat{G}_{d}$ and $\hat{G}_{P} \cup \check{G}_{P}$ are open in $\hat{G}_{r}$, the topology on $\check{G}_{P} \cup \hat{G}_{d}$ is discrete, and the closure of any subset $S \subseteq \hat{G}_{P}$ consists precisely of those $\pi \in \hat{G}_{P} \cup \check{G}_{P}$ which are associated with the points in the natural closure of $S$ in $\mathcal{Q}_{P}$.

3. The structure of $C_{\rho}^{*}(G)$. Since $R=\{\sigma \in \hat{M}: w \cdot \sigma=\sigma\}$ and $I=\hat{M}-R$, we have $\hat{M} \times \hat{A}=(R \times \hat{A}) \cup(I \times \hat{A})$ where both $R \times \hat{A}, I \times \hat{A}$ are $W$-invariant and, in fact, $(R \times \hat{A}) / W=R \times[0, \infty)$. Let $B=[0, \infty)$ and for $\sigma \in R$ write $B_{\sigma}=\{(\sigma, s): s>0\}, \bar{B}_{\sigma}=\{(\sigma, s): s \geqslant 0\}$, and $B_{\sigma}^{\prime}=B_{\sigma} \cup\left\{\pi_{\sigma}^{ \pm}\right\}$. Let

$$
\mathscr{Q}_{R}=R \times B=\bigcup_{\sigma \in R} \bar{B}_{\sigma}, \quad \mathscr{Q}_{I}=(I \times \hat{A}) / W \quad \text { and } \quad B_{\theta}=\mathscr{Q}_{I} \cup \hat{G}_{d} .
$$

Then

$$
\mathscr{2}_{P}=\mathscr{Q}_{R} \cup \mathscr{2}_{I} \quad \text { and } \mathscr{Q}_{P} \cup \hat{G}_{d}=\left(\bigcup_{\sigma \in R} \bar{B}_{\sigma}\right) \cup B_{\theta} .
$$

According to the results of $\S 2$, each of the (Hausdorff) fibres $\bar{B}_{\sigma}$ in $\mathscr{Q}_{R}$ is associated with the (non-Hausdorff) fibre $B_{\sigma}^{\prime}$ in $\hat{G}_{r}$ where the topology on $B_{\sigma}^{\prime}$ is such that as $s \rightarrow 0$ in the usual sense, $(\sigma, s)$ approaches both $\pi_{\sigma}^{ \pm}$as limit points in $\hat{G}_{r}$. Thus we may write $\hat{G}_{r}=\left(\cup_{\sigma \in R} B_{\sigma}^{\prime}\right) \cup B_{\theta}$.

Let $I_{\theta}$ denote the ideal in $C_{\rho}^{*}(G)$ with $\hat{I}_{\theta}=B_{\theta}$ and $I_{\sigma}$ denote the ideal in $C_{\rho}^{*}(G)$ with $\hat{I}_{\sigma}=B_{\sigma}^{\prime}, \sigma \in R$. Let $\mathscr{Q}$ be as in [8, Volume II, p. 50]. Then $\mathscr{Q l}$ is also a dense selfadjoint subalgebra of $C_{\rho}^{*}(G)$ with each element boundedly represented in $\hat{G}_{r}$. Since each $B_{\theta}$ and $B_{\sigma}^{\prime}, \sigma \in R$, is both open and closed in $\hat{G}_{r}$, each $I_{\sigma}, \sigma \in R \cup\{\theta\}$, is a direct summand of $C_{\rho}^{*}(G)$. So for $\sigma \in R \cup$ 
$\{\theta\}$ we may let $\mathscr{U}_{\sigma}$ denote the canonical image of $\mathscr{U}$ in $I_{\sigma}$. Then $\mathscr{U}_{\sigma}$ is a dense selfadjoint subalgebra of $I_{\sigma}$ having the property that each of its elements is boundedly represented in $\hat{I}_{\sigma}$. We now use the extension theory of Delaroche to give concrete descriptions of these ideals and then prove that $C_{\rho}^{*}(G)$ is isomorphic to the restricted product $[2,1.9 .14]$ of these ideals.

Proposition 1. (i) Let $\sigma \in R$. Then $I_{\sigma}$ is isomorphic to the $C^{*}$-algebra of pairs

$$
\left(m,\left(c_{1}, c_{2}\right)\right) \in C^{b}\left(B_{\sigma}, \mathcal{K}(H)\right) \times(\mathscr{K}(H) \oplus \mathscr{K}(H))
$$

such that $\lim _{t \rightarrow \infty} m(\sigma, t)=0$ and $\lim _{t \rightarrow 0} m(\sigma, t)=\left(c_{1}, c_{2}\right)$.

(ii) $I_{\theta}$ is isomorphic to $C^{0}\left(B_{\theta}\right.$, א్K $\left.(H)\right)$.

Proofs. (i) For $\sigma \in R$, let $J_{\sigma}$ be the ideal of $I_{\sigma}$ with $\hat{J}_{\sigma}=B_{\sigma}$. From [8, Volume II, p. 50] it follows that $J_{\sigma}$ is a $C^{*}$-algebra with continuous trace [2, 4.5.2]. Since $H^{3}\left(B_{\sigma}, \mathbf{Z}\right)=0$, it follows from $[2,10.9 .6]$ that $J_{\sigma}$ is isomorphic to $C^{0}\left(B_{\sigma}, \mathfrak{K}(H)\right)$. Now $I_{\sigma}$ is isomorphic to an extension of $C^{0}\left(B_{\sigma}, \mathfrak{K}(H)\right)$ by $\mathcal{K}(H) \oplus \mathcal{K}(H)$, in fact, using [1, Theorem VI.3.8], one can concretely describe $I_{\sigma}$ as above once the positive integers $m$ and $n$ are determined in the equation

$$
\lim _{t \rightarrow 0} \operatorname{tr} \pi(\sigma, t)(f)=m \operatorname{tr} \pi_{\sigma}^{+}(f)+n \operatorname{tr} \pi_{\boldsymbol{\sigma}}^{-}(f), \quad f \in \mathcal{Q l}_{\boldsymbol{\sigma}} .
$$

However, the results of $[8$, Volume II, p. 50] show that $m=n=1$ and so (i) follows.

(ii) Since $\hat{I}_{\theta}$ is Hausdorff and $H^{3}\left(I_{\theta}, \mathbf{Z}\right)=0$, (ii) follows from $[2,10.9 .6]$ since $[8$, Volume II, pp. 50,422$]$ shows that $I_{\theta}$ is a $C^{*}$-algebra with continuous trace.

Proposition 2. Let $\sigma \in R$. Then $I_{\sigma}$ is isomorphic to the subalgebra $D$ of functions in $C^{0}\left(\bar{B}_{\sigma}, \mathcal{K}(H)\right)$ which reduce at $(\sigma, 0)$ by $H \oplus H$.

Proof. For $f \in D$, the pair $\left(m,\left(c_{1}, c_{2}\right)\right)$ where $m(\sigma, t)=f(\sigma, t)$ for $t \in(0$, $\infty)$ and $\left(c_{1}, c_{2}\right)=f(\sigma, 0)$ is clearly in $I_{\sigma}$. Since the mapping $f \mapsto\left(m,\left(c_{1}, c_{2}\right)\right)$ is an isomorphicm of $D$ onto $I_{\sigma}$, the proposition follows.

Lemma 1. Let a be a $C^{*}$-algebra without identity. If $\hat{\mathrm{a}}=\cup_{1}^{\infty} X_{n}$ where the $X_{n}$ are disjoint nonempty open subsets of $\hat{\mathfrak{a}}$, then $\mathfrak{a}$ is isomorphic to the restricted product $B$ of the ideals $I_{n}$, where $\hat{I}_{n}=X_{n}$.

Proof. Let $C=\cup_{k=1}^{\infty} \bigoplus_{n=1}^{k} I_{n}$ and consider the ideal $J=\bar{C}$ of a. It is easy to see that for any $\pi \in \hat{a}, \pi(J) \neq 0$. Thus $J=a$ by [2, 3.2.2]. We now map $C$ onto a dense subset of $B$ in the obvious way. Since this mapping is an isometry, it extends to an isomorphism of a onto $B$.

THEOREM 1. $C_{\rho}^{*}(G)$ is isomorphic to the restricted product of the $C^{*}$-algebras $I_{\sigma}, \sigma \in R \cup\{\theta\}$.

Proof. Since $\widehat{C_{\rho}^{*}(G)}=\hat{G}_{r}=\left(\cup_{\sigma \in R} B_{\sigma}^{\prime}\right) \cup B_{\theta}$, this is immediate from Lemma 1. 
THEOREM 2. $C_{\rho}^{*}(G)$ is isomorphic to the subalgebra of $C^{0}\left(\mathcal{Q}_{P} \cup \hat{G}_{d}, \mathcal{K}(H)\right)$ of functions which reduce at the points of $\mathcal{Q}_{P}-\hat{G}_{P}$ by $H \oplus H$. In particular, when $\check{G}_{P}=\varnothing, C_{\rho}^{*}(G)$ is isomorphic to $C^{0}\left(\hat{G}_{r}, \mathcal{K}(H)\right)$.

Proof. By Theorem 1 we have that $C_{\rho}^{*}(G)$ is isomorphic to the restricted product $P$ of the ideals $I_{\sigma}, \sigma \in R \cup\{\theta\}$ whose structures are given by Propositions 1(ii) and 2. For $f=\left\{f_{\sigma}\right\}$ in $P$ we define the function $F$ on $\mathcal{Q}_{P} \cup \hat{G}_{d}=\left(\cup_{\sigma \in R} \bar{B}_{\sigma}\right) \cup B_{\theta}$ by $F(v)=f_{\sigma}(v)$ if $v \in \bar{B}_{\sigma}, \sigma \in R$, and $F(v)=$ $f_{\theta}(v)$ if $v \in B_{\theta}$. Then $F$ is easily seen to be a norm-continuous bounded function on the Hausdorff space $\mathcal{Q}_{P} \cup \hat{G}_{d}$ for which $\|F(t)\|$ vanishes at infinity and $F(\sigma, 0)=f_{\sigma}(0,0)=\left(c_{1}(\sigma), c_{2}(\sigma)\right)$ for $\sigma \in R$. Theorem 2 now follows since the mapping $f \mapsto F$ is an isomorphism of $P$ onto the above subalgebra.

4. Some examples. A. If $G=\operatorname{Spin}(2 n+1,1)$ for $n \geqslant 1$, then $\check{G}_{P}=\varnothing$ (see [5] or [7]). Since $\hat{G}_{d}=\varnothing$, we have $\hat{G}_{r}=\hat{G}_{P}$ is Hausdorff and

$$
C_{\rho}^{*}(G) \approx C^{0}\left(\hat{G}_{r}, \mathcal{K}(H)\right) \text {. }
$$

B. For $G=\operatorname{SL}(2, \mathbf{R}), M=\{ \pm e\}$ and we may take $\hat{M}=\{0,1\}$ with $R=\{1\}$ and $I=\{0\}$. Thus we may identify $\hat{G}_{r}$ with the following subset of $\mathbf{R}^{2}: \hat{G}_{P}$ consists of the two fibres $\{(0, s): s \geqslant 0\}$ and $\{(1, s): s>0\} ; \check{G}_{P}$ is a pair of points at $\left(1,-\frac{1}{2}\right)$; and $\hat{G}_{d}$ consists of the infinite collection of pairs of points at $(-1,-n), n=1, \frac{3}{2}, 2, \ldots$ The hull-kernel topology on $\hat{G}_{r}$ is then the relative topology $\hat{G}_{r}$ obtains as a subset of $\mathbf{R}^{2}$ with the one exception that as $(1, s) \rightarrow(1,0)$ in the usual sense, $(1, s)$ approaches the pair of points at $(1$, $\left.-\frac{1}{2}\right)$ as limit points. To describe $C_{\rho}^{*}(G)$ we let $X=\{(0, s): s \geqslant 0\} \cup\{(1, s)$ : $s \geqslant 0\} \cup \hat{G}_{d}$ with the relative topology of $\mathbf{R}^{2} . C_{\rho}^{*}(G)$ is then isomorphic to the subalgebra of $C^{0}(X, \mathcal{K}(H))$ consisting of functions which reduce at $(1,0)$ by $H \oplus H$.

C. For $G=\operatorname{Spin}(4,1), M=\operatorname{Spin}(3) \approx \operatorname{SU}(2)$ and we may parameterize $\hat{M}$ by nonnegative half-integers with $R=\left\{\frac{1}{2}, \frac{3}{2}, \ldots\right\}, I=\{0,1,2, \ldots\}$. Using the results of Dixmier [3], we may parameterize $\hat{G}_{d}$ by pairs of points at ( $n$, $-q$ ) where $n=1, \frac{3}{2}, 2, \ldots$ and $q=n, n-1, \ldots, \frac{3}{2}$ or 1 . Thus $\hat{G}_{r}$ can be identified with the following subset of $\mathbf{R}^{2}: \hat{G}_{P}$ is the collection of fibres $\{(n$, $s): s \geqslant 0$ if $n \in I$ and $s>0$ if $n \in R\} ; \check{G}_{P}$ is the infinite collection of pairs of points at $\left(n,-\frac{1}{2}\right), n \in R$; and $\hat{G}_{d}$ consists of the infinite collection of pairs of points at $(n,-q), n=1, \frac{3}{2}, \ldots$ and $q=n, n-1, \ldots, \frac{3}{2}$ or 1 . Since $\check{G}_{P} \neq \varnothing, \hat{G}_{r}$ is not Hausdorff. To describe $C_{\rho}^{*}(G)$ we let $X=\cup_{n \in R \cup I}\{(n, s)$ : $s \geqslant 0\} \cup \hat{G}_{d}$ with the relative topology of $\mathbf{R}^{2}$. Then $C_{\rho}^{*}(G)$ is isomorphic to the subalgebra of $C^{0}(X$, $\mathcal{K}(H))$ consisting of functions which reduce at the points $(n, 0), n \in R$, by $H \oplus H$.

D. Let $G=\mathrm{SO}_{e}(n, 1), n \geqslant 2$, and $G^{\prime}$ be the two-fold covering of $G$-so $G^{\prime}=\operatorname{SL}(2, \mathbf{R})$ for $n=2$ and $G^{\prime}=\operatorname{Spin}(n, 1)$ for $n \geqslant 3$. $G^{\prime}$ then satisfies the hypotheses of this paper. From [5] we know that even though $G^{\prime}$ may have reducible principal series (iff $n$ is even), $G$ does not. Since $\hat{G}_{r} \subseteq \hat{G}_{r}^{\prime}$ has the relative hull-kernel topology, we see that $\hat{G}_{r}$ is Hausdorff [for example, if 
$n=2$ and $D$ denotes the subset of $\hat{G}_{d}($ in $B)$ consisting of pairs of points at $(-1,-n), n=1,2, \ldots$, then $\hat{G}_{r}=\{(0, s): s \geqslant 0\} \cup D$, while if $n=4$ and $D$ denotes the subset of $\hat{G}_{d}$ (in $C$ ) of pairs of points at $(n,-q), n \in I$, then $\left.\hat{G}_{r}=\cup_{n \in I}\{(n, s): s \geqslant 0\} \cup D\right]$. Thus it follows, as in the proof of Proposition $1\left(\right.$ ii), that $C_{\rho}^{*}(G) \approx C^{0}\left(\hat{G}_{r}, \mathcal{K}(H)\right)$.

5. A remark on $C_{0}(\hat{G})$. When $G$ is a locally compact abelian group, it is common to denote the collection of continuous functions on the dual group $\hat{G}$ which vanish at infinity by $C_{0}(\hat{G})$. In a recent paper [6], R. Lipsman defined an analogue of this space for separable locally compact unimodular type I groups as follows: letting $d g$ denote Haar measure on $G$,

$$
\mathscr{F} f(\pi)=\hat{f}(\pi)=\int f(g) \pi(g) d g
$$

be the Fourier-transform of $f \in L_{1}(G),\||| \hat{f}(\pi) \mid\|$ the operator norm of $\hat{f}(\pi)$, $\|\hat{f}\|_{\infty}=\operatorname{ess} \sup _{\pi \in \hat{G}}\|\hat{f}(\pi)\| \|$ (with respect to Plancherel measure on $\hat{G}$ ), and $A(\hat{G})=\mathscr{\mathscr { Y }}\left(L_{1}(G)\right)$, then $C_{0}(\hat{G})$ is defined to be the closure of the algebra $A(\hat{G})$ with respect to the norm $\|\cdot\|_{\infty}$. The question is then raised as to determining an intrinsic characterization of $C_{0}(\hat{G})$. Since $C_{0}(\hat{G})$ is easily seen to be isomorphic to $C_{\rho}^{*}(G)$ (see [6]), the results of this paper seem to indicate that this will be a difficult problem and that the hull-kernel topology on the spectrum of $C_{\rho}^{*}(G), \hat{G}_{r}$, will play a key role in determining an intrinsic characterization of $C_{0}(\hat{G})$. In fact, Theorem 2 shows that when $G$ is as in the introduction of this paper, an intrinsic characterization of $C_{0}(\hat{G})$ must take the non-Hausdorff nature of $\hat{G}_{r}$ into consideration.

We also remark that for amenable groups, $C_{0}(\hat{G}) \approx C_{\rho}^{*}(G) \approx C^{*}(G)$ (the group $C^{*}$-algebra of $\left.G\right)$, and although it is quite easy to describe $C_{0}(\hat{G})$ for abelian or compact groups, we know of no other separable unimodular type I amenable group for which the structure of $C^{*}(G)$ has been determined.

\section{REFERENCES}

1. C. Delaroche, Extensions des $C^{*}$-algèbres, Bull. Soc. Math. France, Mémoire 29, 1972.

2. J. Dixmier, Les $C^{*}$-algèbres et leurs représentations, 2nd ed., Gauthier-Villars, Paris, 1969.

3. , Représentations intégrables du groupe de De Sitter, Bull. Soc. Math. France 89 (1961), 9-41. MR 25 \#4031.

4. A. W. Knapp, Commutativity of intertwining operators, Bull. Amer. Math. Soc. 79 (1973), 1016-1018. MR 48 \#11399.

5. A. W. Knapp and E. M. Stein, Intertwining operators for semisimple groups, Ann. of Math. (2) 93 (1971), 489-578.

6. R. L. Lipsman, Non-abelian Fourier analysis, Bull. Sci. Math. 98 (1974), 209-233.

7. N. R. Wallach, Cyclic vectors and irreducibility for principal series representations, Trans. Amer. Math. Soc. 158 (1971), 107-113. MR 43 \# 7558.

8. G. Warner, Harmonic analysis on semisimple Lie groups. Vols. I, II, Springer-Verlag, Berlin, 1972.

Department of Mathematics, University of Pennsylvania, Philadelphia, Pennsylvania 19174 (Current address of Robert Boyer)

Current address (Robert Martin): Department of Mathematics, Middlebury College, Middlebury, Vermont 05753 